\title{
Reverse remodeling is associated with changes in extracellular matrix proteases and tissue inhibitors after mesenchymal stem cell (MSC) treatment of pressure overload hypertrophy
}

\author{
Ezequiel J. Molina ${ }^{1}$, Jon Palma ${ }^{1}$, Dipin Gupta ${ }^{1}$, Denise Torres ${ }^{1}$, John P. Gaughan ${ }^{2}$, Steven Houser ${ }^{2}$ \\ and Mahender Macha ${ }^{3 *}$ \\ ${ }^{1}$ Division of Cardiac and Thoracic Surgery, Temple University School of Medicine, Philadelphia, PA, USA \\ ${ }^{2}$ Department of Physiology, Temple University School of Medicine, Philadelphia, PA, USA \\ ${ }^{3}$ Division of Cardiac Surgery, University of Michigan Medical School, Ann Arbor, MI, USA
}

\begin{abstract}
Changes in ventricular extracellular matrix (ECM) composition of pressure overload hypertrophy determine clinical outcomes. The effects of mesenchymal stem cell (MSC) transplantation upon determinants of ECM composition in pressure overload hypertrophy have not been studied. Sprague-Dawley rats underwent aortic banding and were followed by echocardiography. After an absolute decrease in fractional shortening of $25 \%$ from baseline, $1 \times 10^{6}$ MSC $(n=28)$ or PBS $(n=20)$ was randomly injected intracoronarily. LV protein analysis, including matrix metalloproteinases (MMP-2, MMP-3, MMP-6, MMP-9) and tissue inhibitors of metalloproteinases (TIMP-1, TIMP-2, TIMP-3), was performed after sacrifice on postoperative day 7, 14, 21 or 28 . Left ventricular levels of MMP-3, MMP-6, MMP-9, TIMP-1 and TIMP-3 were demonstrated to be decreased in the MSC group compared with controls after 28 days. Expression of MMP-2 and TIMP-2 remained relatively stable in both groups. Successful MSCs delivery was confirmed by histological analysis and visualization of labelled MSCs. In this model of pressure overload hypertrophy, intracoronary delivery of MSCs during heart failure was associated with specific changes in determinants of ECM composition. LV reverse remodeling was associated with decreased ventricular levels of MMP-3, MMP-6, MMP-9, TIMP-1 and TIMP-3, which were upregulated in the control group as heart failure progressed. These effects were most significant at 28 days following injection. Copyright $\odot 2008$ John Wiley \& Sons, Ltd.
\end{abstract}

Received 3 February 2008; Revised 12 September 2008; Accepted 25 September 2008

Keywords pressure overload hypertrophy; heart failure; stem cell therapy; mesenchymal stem cells; cardiac remodeling; extracellular matrix

\section{Introduction}

Cardiac remodeling is a major determinant of the clinical course of congestive heart failure (CHF), irrespective of its aetiology (Cohn et al., 2000; Fedak et al., 2005a; Udelson and Konstam, 2002). New approaches that promote reverse cardiac remodeling are being evaluated.

*Correspondence to: Mahender Macha, Suite 301, 1100 E. Michigan Avenue, Jackson, MI 49201, USA.

E-mail: mmacha@med.umich.edu
Among these alternative approaches, cellular transplantation therapy has emerged with particular relevance. Several animal studies have shown that the implantation of different populations of cells in failing myocardium has a profound and consistent anti-remodeling effect that limits progression to heart failure (Li et al., 1996, 1999, 2000a; Nagaya et al., 2005; Rajnoch et al., 2001; Saito et al., 2003; Silva et al., 2005; Suzuki et al., 2001; Tomita et al., 2002). However, most of these studies have been performed in models of ischaemic cardiomyopathy. Despite the high prevalence of chronic hypertension in 
Western countries, few studies have shown the effects of cell transplantation therapy in models of pressure overload hypertrophy (Wang et al., 2006).

The extracellular matrix (ECM) provides the structural support for cardiac cells but also serves as a dynamic microenvironment for cell signalling within the extracellular space (Lukashev and Werb, 1998). ECM remodeling is now recognized as a central process underlying the maladaptive reorganization of cardiac size, shape and function during the progression of CHF. Extracellular matrix remodeling is thought to be determined by the balance of degradative enzymes, the metalloproteinases (MMPs), with respect to highly regulated and complex endogenous inhibitors, the tissue inhibitors of metalloproteinases (TIMPs) (Fedak et al., 2005b).

In a rat model of pressure overload hypertrophy, we have recently found an improvement in systolic and diastolic function, prolonged exercise tolerance, amelioration of the inflammatory profile and echocardiographic signs of reverse left ventricular remodeling after intracoronary delivery of bone marrow-derived mesenchymal stem cells (MSCs; Molina et al., 2008). In the present study, we hypothesized that reverse LV remodeling in this model is associated with specific changes in ventricular expression of MMPs and TIMPs during the progression of heart failure.

\section{Materials and methods}

All animals received humane care in compliance with the animal care and use guidelines of the Institutional Animal Care and Use Committee (IACUC) of Temple University School of Medicine. All procedures conformed to the Principles of Laboratory Animal Care (NIH Publication No. 86-23, revised 1985).

\subsection{Experimental model of heart failure}

Male Sprague-Dawley rats (200-250 g; Harlan, Indianapolis, IN, USA) were anaesthetized, endotracheally intubated and mechanically ventilated. An upper median sternotomy was performed and a small titanium clip with an internal diameter of $0.6 \mathrm{~mm}$ (Ligaclip ${ }^{\circledR}$ Extra, Ethicon Endo-Surgery Inc., Cincinnati, OH, USA) was applied across the aorta, proximal to the right brachiocephalic artery. All animals underwent baseline echocardiography 10 weeks after clip placement. Transthoracic M-mode and two-dimensional (2D) echocardiography was performed using a Hewlett-Packard Sonos 5500 Imaging System with a $4 \mathrm{MHz}$ transducer. Images were standardized to short axis view at the left ventricular mid-papillary level and fractional shortening (FS), end-systolic diameter (LVESD), end-diastolic diameter (LVEDD) and left ventricular free wall diameter (FWD) were recorded. Starting at 10 weeks after aortic clip placement, echocardiography was performed weekly. Following detection of a decline in fractional shortening of $25 \%$ from baseline, the animals were randomized to intracoronary delivery of bone marrow-derived MSCs (MSC group, $n=28$ ), or phosphate-buffered saline (PBS; control group, $n=20$ ). Following treatment with MSCs or PBS, animals were randomly sacrificed on post-operative day 7, 14, 21 or 28. Serial echocardiography was performed weekly to identify reverse remodeling.

\subsection{Isolation of bone marrow-derived MSCs and culture technique}

MSCs were kindly provided by Dr S Ausim Azizi (Neurology Department, Temple University School of Medicine). Primary cultures of MSCs were obtained from the femurs and tibias of adult male Sprague-Dawley rats (Taconic Farms, Germantown, NY, USA) as described previously (Molina et al., 2008; Schwarz et al., 2001). Briefly, rats were euthanized with a mixture of $70 \%$ $\mathrm{CO}_{2}$ and $30 \% \mathrm{O}_{2}$. Tibias and femurs were removed and placed on ice in complete medium containing minimal essential medium (MEM) with alpha modification ( $\alpha$ MEM; Gibco-BRL, Gaithersburg, MD, USA) with $20 \%$ fetal calf serum (Atlanta Biologicals, Norcross, GA, USA), $2 \mathrm{~mm}$ L-glutamine, penicillin (100 units $/ \mathrm{ml}$ ), streptomycin $(100 \mu \mathrm{g} / \mathrm{ml})$, and amphotericin B (25 ng/ml; Mediatech, Herndon, VA). Under sterile conditions, a 21 gauge needle attached to a $10 \mathrm{ml}$ syringe filled with medium was used to flush out the marrow. Bone marrow was filtered through a $70 \mu \mathrm{m}$ nylon mesh and plated in a $75 \mathrm{~cm}^{2}$ flask (Becton-Dickinson, Franklin Lakes, NJ, USA). MSCs were isolated by their adherence to plastic. About $24 \mathrm{~h}$ after plating, non-adherent cells were removed and fresh medium was added. After the cells had grown to near-confluency, they were passaged two to six times by being detached ( $0.25 \%$ trypsin/ $1 \mathrm{~mm}$ EDTA for $5 \mathrm{~min}$ ) and replated at a density of around 5000 cells $/ \mathrm{cm}^{2}$ (high-density conditions). At this point the cells were frozen in vials containing approximately 500000 cells per unit. Preliminary studies with RT-PCR revealed that these cells expressed GATA-4 and Nkx2.5, which are early cardiac lineage markers (data not shown). Five days before intracoronary cell delivery in rats, the MSCs were thawed and cultured in 10\% $\alpha$-MEM for $24 \mathrm{~h}$. The second day, MSCs were plated and 10\% Iscove's Modified Dulbecco's Media (IMDM) fresh medium was added. In addition, bromodeoxyuridine (BrdU) was supplemented to the culture medium at a concentration of $5 \mu \mathrm{M}$ for $24 \mathrm{~h}$. The efficacy of BrdU labelling approached 100\%, as observed by light microscopy. No signs of cell toxicity were observed. On the third day, 5-azacytidine was added to the culture medium at a concentration of $10 \mu \mathrm{g} / \mathrm{ml}$ for $24 \mathrm{~h}$. The demethylating agent 5-azacytidine has been shown to induce cardiomyocyte differentiation in bone marrow-derived MSCs in vitro (Makino et al., 1999). Culture medium was then replaced with fresh $10 \%$ IMDM on the fourth day. Finally, on the fifth day, MSCs were trypsinized and were then ready for injection. Solution $(150 \mu \mathrm{l})$ containing $1 \times 10^{6} \mathrm{MSCs}$ in PBS, or 
PBS alone, was mixed with $50 \mu \mathrm{l}$ adenosine $(3 \mathrm{mg} / \mathrm{ml}$; Fujisawa Healthcare Inc., Deerfield, IL, USA) just prior to injection. We chose this particular cell dose based on prior studies of MSC and myoblast transplantation, which have demonstrated the safety and efficacy of intracoronary infusion of 1 million cells (Saito et al., 2003; Suzuki et al., 2001). Although we do not have data to elucidate the effects of adenosine upon cell retention, it is possible that the vasodilatory properties of this drug could increase intracoronary cell delivery and myocardial cell retention in this experimental model.

\subsection{MSC delivery}

Within $48 \mathrm{~h}$ of detection of an absolute $25 \%$ decline in fractional shortening from baseline and subsequent randomization, the animals were anaesthetized and a full median sternotomy was performed. The aorta and main pulmonary artery were encircled together with 4-0 prolene and a tourniquet was loosely applied. Solution $(150 \mu \mathrm{l})$ containing $1 \times 10^{6}$ MSCs in PBS, or PBS alone, was mixed with $50 \mu \mathrm{l}$ adenosine just prior to injection. Using a $1 \mathrm{ml}$ syringe attached to a sterile 30 gauge needle, the solution was injected into the aortic root during brief (5 s) occlusion of the aorta and the pulmonary artery. Intracoronary delivery was confirmed intraoperatively by temporary observation of bradycardia and epicardial blanching.

\subsection{Protein assay and western blot analysis}

Protein analysis, including LV myocardial levels of MMP-2, MMP-3, MMP-6, MMP-9, TIMP-1, TIMP-2 and TIMP-3 was performed. We adhered to standard protein assay techniques, which have been published previously (Bristow, 2002). Tissue samples underwent electrophoresis on 8-20\% SDS-polyacrylamide gels (SDS-PAGE) after $30 \mathrm{~min}-1 \mathrm{~h}$ of denaturation at $37^{\circ} \mathrm{C}$. Proteins were then transferred to Immobilon$\mathrm{P}$ (Millipore) by use of wet transfer apparatus. To prevent non-specific binding, the membrane was blocked using 5\% non-fat dried milk in Tris-buffered saline (0.1\% Tween 20, $137 \mathrm{~mm} / \mathrm{l} \mathrm{NaCl}, 2.7 \mathrm{mM} / \mathrm{l} \mathrm{KCl}$, and $25 \mathrm{~mm} / 1$ Tris base, $\mathrm{pH}$ 7.6). Antigens of interest were probed with the following polyclonal antibodies: MMP-2, MMP-3, MMP-6, MMP-9, TIMP-1, TIMP-2 and TIMP-3 (Santa Cruz Biotechnology, Santa Cruz, CA, USA). Detection was performed using enhanced chemiluminescence assay. The Immobilon-P membrane was stained (Coomassie blue) and the actin band was identified, thus confirming equal loading conditions. Films were scanned and band intensities were quantified by densitometric analysis by the use of the NIH Image $1.62 \mathrm{f}$ programme. All targeted bands were normalized to cardiac actin.

\subsection{Histological analysis}

Additional animals were utilized to confirm successful delivery and survival of MSCs after intracoronary delivery. Animals were euthanized by cardiectomy 2 h, 7 days and 21 days after treatment and the heart was thoroughly washed with PBS cooled to $4^{\circ} \mathrm{C}$. After resection of the atria, the ventricles were fixed in $10 \%$ formalin for $24 \mathrm{~h}$. Hearts were embedded in paraffin and cut into slices $4 \mu \mathrm{m}$ thick. Monoclonal antibodies against BrdU (BrdU staining kit, Zymed, San Francisco, CA, USA) were used to identify transplanted cells. For each time point, 10 separate heart sections were examined under $\times 400$ magnification, using a E600 Nikon microscope.

\subsection{Statistical analysis}

All data are presented as mean \pm standard deviation (SD). Between-group differences in frequency data were analysed using Fisher's exact test. Differences in continuous variables were analysed using independent $t$ tests. Analysis of variance (ANOVA) with least significant difference (LSD) procedure was used. After ANOVA, a Bonferroni post hoc analysis was used for adjustment for multiple comparisons. The SAS v. 8.1 (SAS Institute Inc., Cary, NC, USA) was used for statistical analysis. $p<0.05$ was considered to be statistically significant. The authors had full access to the data and take responsibility for its integrity. All authors have read and agree to the manuscript as written.

\section{Results}

\subsection{Induction of heart failure}

Most animals reached an absolute decline in fractional shortening of $25 \%$ compared to baseline 28-30 weeks after aortic clip placement (mean \pm SD, $203 \pm 15$ days). Fractional shortening was similar between animals in the MSC and control groups at baseline echocardiographic assessment ( $51.2 \pm 1.7 \%$ vs. $51.0 \pm 2.2 \% ; p=0.71)$ and treatment randomization $(26.5 \pm 1.7 \%$ vs. $26.0 \pm 2.1 \%$; $p=0.41$ ). We observed a combined $30 \%$ mortality rate during aortic clip placement and development of heart failure. Four additional animals died after treatment (three in the control group and one in the MSC group).

\subsection{Expression of metalloproteinases}

Table 1 shows changes in left ventricular expression of metalloproteinases in both groups at different time intervals. Representative western blot images are shown in Figure 1 (28 days only). Left ventricular expression of MMP-2 was found to be relatively stable, with progression of heart failure in both groups. Although expression of this marker in cell-treated animals was lower than in controls 
Table 1. Left ventricular levels of metalloproteinases and tissue inhibitors of metalloproteinases (level/actin) at 7, 14, 21 and 28 days after treatment

\begin{tabular}{|c|c|c|c|c|c|c|c|c|}
\hline & \multicolumn{2}{|c|}{7 Days } & \multicolumn{2}{|c|}{14 Days } & \multicolumn{2}{|c|}{21 Days } & \multicolumn{2}{|c|}{28 Days } \\
\hline & Control & MSC & Control & MSC & Control & MSC & Control & MSC \\
\hline MMP-2 & $1.119 \pm 0.106$ & $1.074 \pm 0.028$ & $1.114 \pm 0.085$ & $1.047 \pm 0.031$ & $1.103 \pm 0.056$ & $1.064 \pm 0.063$ & $1.113 \pm 0.044$ & $1.047 \pm 0.062$ \\
\hline MMP-3 & $1.188 \pm 0.161$ & $1.179 \pm 0.168$ & $1.327 \pm 0.105$ & $1.234 \pm 0.129$ & $1.384 \pm 0.048$ & $1.148 \pm 0.057^{*}$ & $1.538 \pm 0.086$ & $1.090 \pm 0.108^{*}$ \\
\hline MMP-6 & $0.999 \pm 0.014$ & $0.995 \pm 0.009$ & $1.006 \pm 0.007$ & $1.001 \pm 0.004$ & $1.005 \pm 0.003$ & $0.996 \pm 0.004$ & $1.016 \pm 0.003$ & $0.986 \pm 0.011^{*}$ \\
\hline MMP-9 & $1.005 \pm 0.023$ & $1.006 \pm 0.024$ & $1.024 \pm 0.004$ & $1.016 \pm 0.013$ & $1.025 \pm 0.003$ & $1.005 \pm 0.008^{*}$ & $1.039 \pm 0.007$ & $0.989 \pm 0.017^{*}$ \\
\hline TIMP-1 & $1.005 \pm 0.023$ & $1.000 \pm 0.016$ & $1.018 \pm 0.014$ & $1.009 \pm 0.009$ & $1.019 \pm 0.004$ & $1.001 \pm 0.005^{*}$ & $1.050 \pm 0.007$ & $0.993 \pm 0.011^{*}$ \\
\hline TIMP-2 & $1.005 \pm 0.003$ & $1.005 \pm 0.006$ & $1.004 \pm 0.004$ & $1.002 \pm 0.005$ & $1.004 \pm 0.003$ & $1.003 \pm 0.004$ & $1.002 \pm 0.004$ & $1.001 \pm 0.003$ \\
\hline TIMP-3 & $1.009 \pm 0.010$ & $1.005 \pm 0.009$ & $1.016 \pm 0.004$ & $1.012 \pm 0.006$ & $1.018 \pm 0.002$ & $1.006 \pm 0.004^{*}$ & $1.024 \pm 0.003$ & $1.002 \pm 0.007^{*}$ \\
\hline
\end{tabular}

${ }^{*} p<0.01$ for comparison of control group vs.mMesenchymal stem cell group.

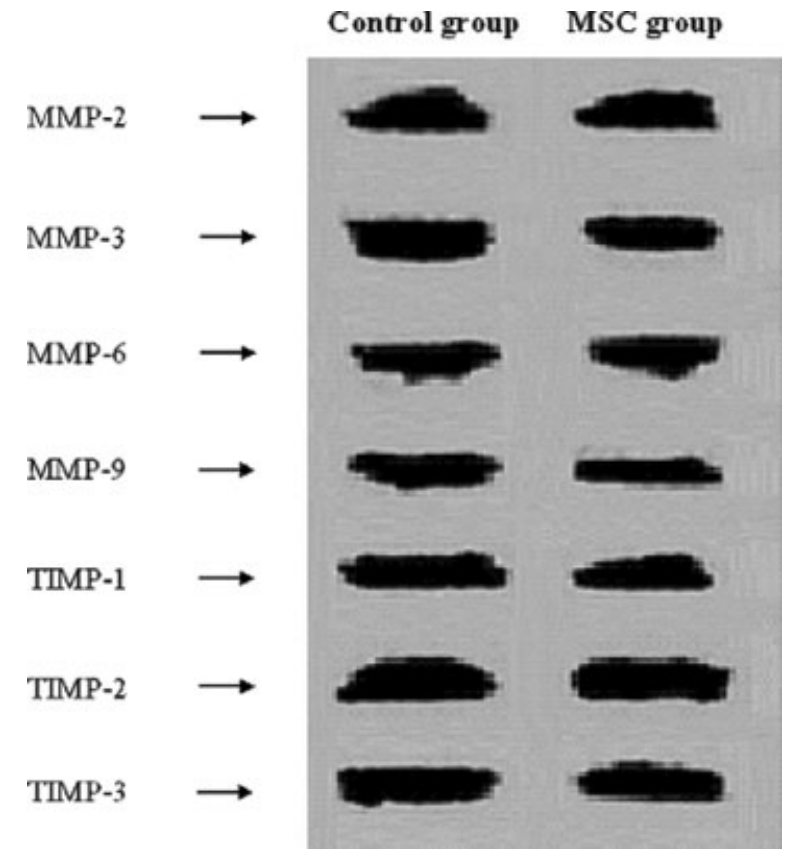

Figure 1. Western blot images showing MMP and TIMP levels 28 days after treatment

at all time points, these differences were not statistically significant. The left ventricular expression of MMP-3 was found to rise with progression of heart failure in the control group. In the cell treatment group, the expression of this enzyme was found to stabilize and slightly decrease after treatment. The differences between groups were statistically significant at days 21 and 28. The expression of MMP- 6 continued to rise with progression of heart failure in the control group, while it was found to decline in the cell treatment group at 28 days. Similarly, MMP-9 expression was found to increase as heart failure progressed in the control group. In the cell treatment group, the expression of this marker declined by 21 and 28 days.

\subsection{Expression of tissue inhibitors of metalloproteinases}

Changes in left ventricular expression of tissue inhibitors of metalloproteinases are shown in Table 1. Representative western blot images are shown in Figure 1 (28 days only). The expression of TIMP-1 increased in the control group as heart failure progressed. The animals that received stem cells revealed an initial increase of the expression of this inhibitor and a subsequent decline. The difference was statistically significant at 28 days. Expression of TIMP-2 remained relatively stable in both groups without significant differences between them. Finally, changes in the expression of TIMP-3 were similar to those of TIMP-1. We observed an initial progressive increase in both groups, followed by a continued rise in control animals and a reduction in treated animals. The differences were again significant at 21 and 28 days.

\subsection{Echocardiographic data}

Left ventricular fractional shortening was found to decline in the control group with progression of heart failure, whereas it remained relatively stable in the MSC treatment group $(25.7 \pm 2.0$ vs. $26.2 \pm 1.8$ at 7 days, $25.3 \pm 2.1$ vs. $26.1 \pm 1.7$ at 14 days, $24.6 \pm 1.9$ vs. $26.4 \pm 1.6$ at 21 days, and $24.1 \pm 1.7$ vs. $26.7 \pm 1.5$ at 28 days; $p<0.01$ at 28 days). Similarly, the control group demonstrated increasing left ventricular end systolic diameters as heart failure progressed, while this diameter remained relatively stable in the MSC group (7.12 \pm 0.3 vs. $7.08 \pm 0.2 \mathrm{~mm}$ at 7 days, $7.17 \pm 0.2$ vs. $7.10 \pm 0.2 \mathrm{~mm}$ at 14 days, $7.25 \pm 0.2$ vs. $7.06 \pm 0.1 \mathrm{~mm}$ at 21 days, and $7.30 \pm 0.1$ vs. $7.03 \pm 0.1 \mathrm{~mm}$ at 28 days; $p=0.01$ at 28 days).

\subsection{Histology}

Representative sections of left ventricular myocardium at different time intervals after intracoronary delivery of MSC were seen by light microscopy. At $2 \mathrm{~h}$, numerous BrdU-labelled MSCs (brown nuclei) are seen among native cardiac cells (blue nuclei). Several mesenchymal cells are visualized in the lumen and around a capillary vessel on the left side of the image. MSC nuclei are rounded, typical of MSCs in culture (Figure 2). A lower magnification view at the same time interval demonstrates an homogeneous distribution of BrdU-stained cells across the ventricular wall (Figure 3). At 7 days, numerous brown-stained cells are visualized among native 


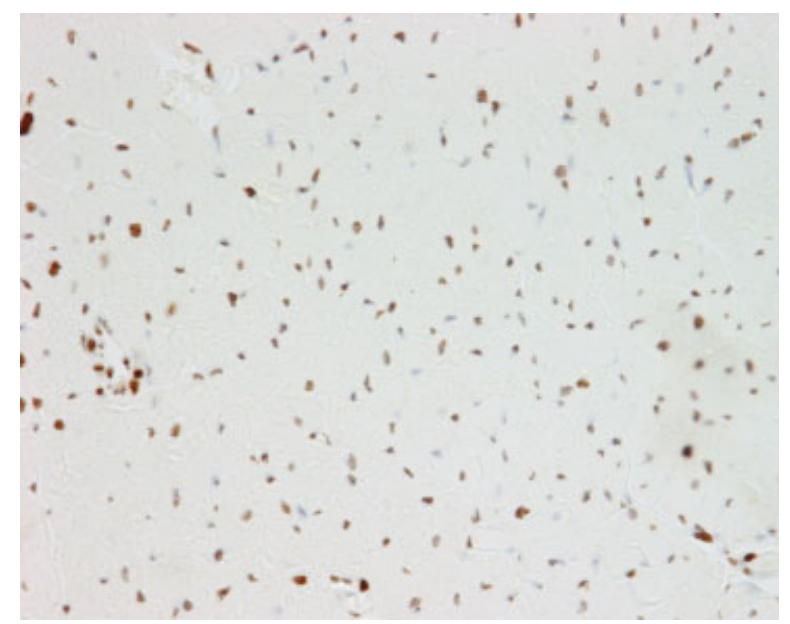

Figure 2. Representative section of LV myocardium, showing BrdU-stained bone marrow-derived mesenchymal stem cells (brown nuclei) among native cardiac cells (blue nuclei), $2 \mathrm{~h}$ following intracoronary delivery $(x 400)$

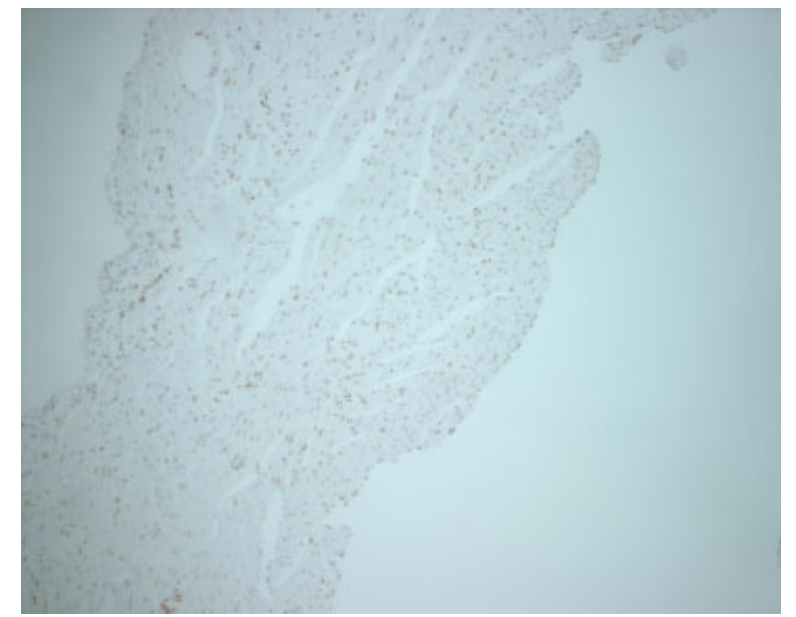

Figure 3. Lower magnification view 2 h after cell transplantation $(\times 100)$

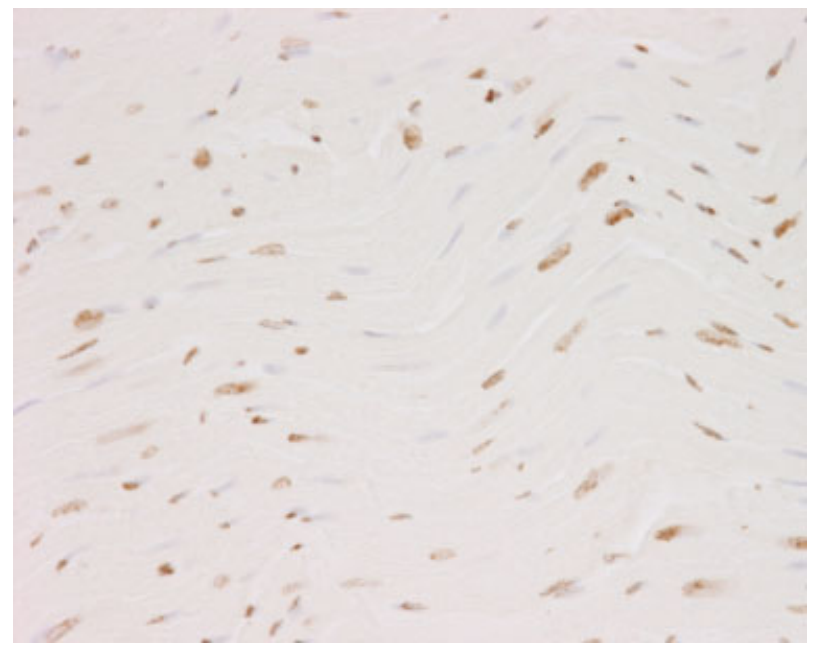

Figure 4. Left ventricular section 7 days following stem cell transplantation $(x 400)$

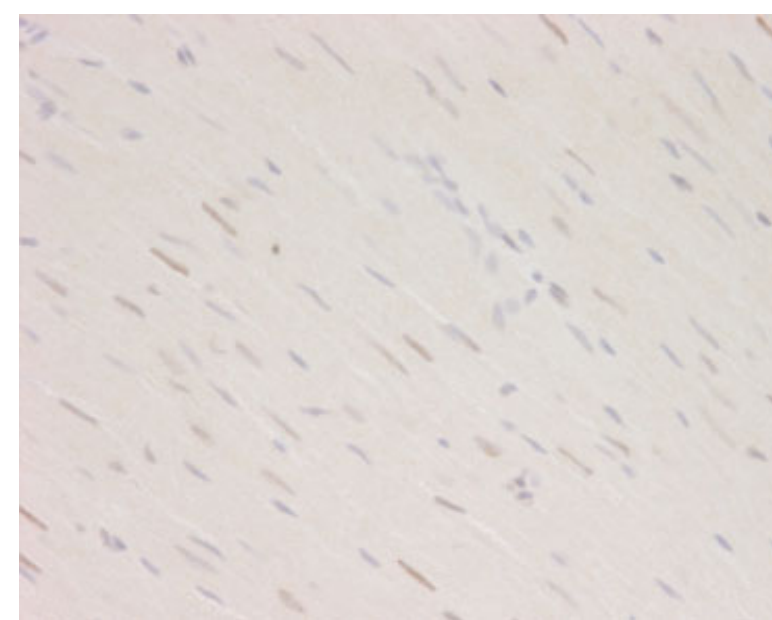

Figure 5. Left ventricular section 21 days following stem cell transplantation $(x 400)$

cardiomyocytes (Figure 4). Some of the brown-stained nuclei are elongated, characteristic of native cardiomyocytes, and some nuclei are rounded. At 21 days after intracoronary delivery, brown-stained nuclei are still visualized in a left ventricular section (Figure 5). The number of stained nuclei and the intensity of the staining has decreased compared with earlier images. Morphologically, most of the BrdU-stained nuclei are elongated.

\section{Discussion}

Although some of the mechanisms underlying cardiac remodeling in ischaemic and non-ischaemic models may be similar, the specific profiles of MMPs and TIMPs during the progression of different forms of heart failure is inconsistent and largely unclear (Fedak et al., 2005b). In patients with ischaemic and non-ischaemic dilated cardiomyopathy, Spinale et al. (1998) reported upregulation of MMP-9 and decreased expression of MMP1. However, MMP-2 and MMP-3 expression was only elevated with non-ischaemic dilated cardiomyopathy. In a cohort of patients post myocardial infarction, Webb et al. (2006) demonstrated long-term upregulation of MMP-9, TIMP-1 and TIMP-2, but not of MMP-2, compared with normal controls. During heart failure progression after myocardial infarction in rats, Peterson et al. (2000) showed increased ventricular expression of MMP-2, MMP-8, MMP-9, TIMP-1 and TIMP-2. In a small cohort of patients with idiopathic dilated cardiomyopathy, Thomas et al. (1998) reported decreased levels of MMP1 and upregulation of MMP-3, MMP-9, TIMP-1 and TIMP-2. In patients with heart failure secondary to aortic stenosis, Polyakova et al. (2004) demonstrated upregulation of MMP-1, MMP-2, MMP-3, MMP-9, MMP13, TIMP-1 and TIMP-2 in comparison with normal controls. In a similar study, however, Fielitz et al. (2004) reported upregulation of MMP-2, TIMP-2 and TIMP-3 and downregulation of MMP-9 and TIMP-1 in the aortic stenosis group. A recent study by Ahmed 
et al. (2006) demonstrated significantly increased MMP9 plasma levels in patients with hypertension and left ventricular hypertrophy compared with healthy controls. Also, hypertensive patients who progressed to heart failure demonstrated increased TIMP-1 plasma levels. In spontaneously hypertensive heart failure rats, Li et al. (2000b) found increased activity of MMP-2, MMP-9, MMP-13, TIMP-1 and TIMP-2. In an experimental model of compensated pressure overload hypertrophy in sheep, upregulation of MMP-1, MMP-2, MMP-3, MMP-9, TIMP1 and TIMP-2 was demonstrated. Interestingly, these changes reversed after surgical release of aortic banding (Walther et al., 2001). Whether this conflicting evidence represents an alternative role of these enzymes in different forms of heart failure, or whether a selective temporal activation occurs, is still unknown.

In this pressure overload model of heart failure, we have recently found an improvement in contractile function, prolonged exercise tolerance, amelioration of inflammatory profile and LV reverse remodeling following intracoronary administration of MSCs (Molina et al., 2008). In this study, we report the associated changes in ECM proteases and tissue inhibitors. We observed upregulation of MMP-3, MMP-6 and MMP9 expression in control animals with progression of heart failure. Similarly, TIMP-1 and TIMP-3 expression was also found to be increased. MMP-2 and TIMP-2 levels, however, remained relatively stable. These results reproduced the findings more consistently reported in the literature in ischaemic and non-ischaemic models of heart failure: upregulation in expression of MMP3, MMP-9 and TIMP-1 with progression of heart failure and LV dilatation (Ahmed et al., 2006; Li et al., 2000b; Peterson et al., 2000; Polyakova et al., 2004; Spinale et al., 1998; Thomas et al., 1998; Webb et al., 2006). Remarkably, animals receiving MSCs showed stabilization or decreased expression of these markers. Left ventricular expression of MMP-3, a metalloproteinase with a wide range of substrates and also a potent activator of other MMPs, was found to decline by 30\% in cell-treated animals at 28 days. Expression of MMP9 , an inducible metalloproteinase whose plasma levels have been found to correlate with LV volumes and ejection fractions in patients with heart failure (Yan et al., 2006), also decreased with cell therapy. The MSC group also demonstrated significantly decreased levels of TIMP-1 by 21 and 28 days after treatment. Although it is not clear whether there is a relationship between myocardial expression and plasma levels of metalloproteinases and their inhibitors, it is interesting to note that increased plasma levels of TIMP-1 have been shown to predict CHF in hypertensive patients with left ventricular hypertrophy (Ahmed et al., 2006). Also, high plasma levels of TIMP-1 have been found to predict mortality and myocardial infarction in a cohort of patients undergoing cardiac angiography (Cavusoglu et al., 2006). The improvement in MMP/TIMP profile and the amelioration of echocardiographic signs of ventricular dilatation and fractional shortening suggest a beneficial role of cell transplantation therapy upon reverse remodeling in this experimental model of heart failure.

Successful stem cell delivery was confirmed in this study by histological analysis of left ventricular myocardium and visualization of labelled MSC immediately after transplantation. Furthermore, viable BrdU-stained nuclei were visualized 21 days after delivery. Whether these cells represent undifferentiated MSCs or differentiated cardiomyocytes is unclear. We plan to perform further immunohistochemical and genetic studies that may help to elucidate this important issue. However, these findings suggest that a significant proportion of transplanted cells may survive and play an important biological role when most of the functional and molecular beneficial effects we reported are manifested.

As stated previously (Fedak et al., 2005c), cell transplantation therapy may promote reverse cardiac remodeling by regulating the MMP-TIMP axis, the central mechanism that controls matrix remodeling in the failing heart. The exact nature of the mechanisms underlying these potential regulatory actions is currently unknown. It is appealing to hypothesize that these biological actions could be mediated by the release of growth factors. These biological mediators could potentially act upon native cardiomyocytes, fibroblasts and/or inflammatory cells, favouring the preservation of ECM homeostasis. Stem cell-mediated downregulation of $\mathrm{TNF} \alpha$ and IL1 local expression, cytokines known to promote the release of MMP-9 and MMP-13 by myocardial fibroblasts (Siwik et al., 2000), could restore normal MMP and TIMP levels in the remodeling myocardium. Engrafted cells could also modulate additional known mechanisms of control of MMP expression, such as the renin-angiotensin-aldosterone system, endothelin-1, the sympathetic system and pathways of oxidative stress (Deschamps and Spinale, 2006). However, these theories remain speculative.

In conclusion, in this model of pressure overload hypertrophy, intracoronary delivery of MSCs during heart failure improved left ventricular reverse remodeling by 28 days. These echocardiographic findings were associated with decreased ventricular levels of MMP3, MMP-6, MMP-9, TIMP-1 and TIMP-3, which were upregulated in the control group as heart failure progressed. These effects were most remarkable at 28 days following injection. Although we acknowledge the preliminary nature of this experimental study, our findings suggest a potential mechanism of MSC therapy in promoting reverse remodeling and myocardial recovery in models of pressure overload hypertrophy. We strongly believe that additional studies of cellular therapy are needed in this model of heart failure, given the high prevalence of hypertensive heart disease in Western countries. Future experimental studies should focus in the mechanisms of action of MSCs and the processes that regulate ECM remodeling. 


\section{Acknowledgements}

We would like to express sincere gratitude to Dr S. Azizi, Dr B. Krynska and B. Augelli (Neurology Department, Temple School of Medicine) for providing mesenchymal stem cells and continuous technical support throughout this study.

\section{References}

Ahmed SH, Clark LL, Pennington WR, et al. 2006; Matrix metalloproteinases/tissue inhibitors of metalloproteinases: relationship between changes in proteolytic determinants of matrix composition and structural, functional, and clinical manifestations of hypertensive heart disease. Circulation 113: 2089-2096.

Bristow MR. 2002; $\beta$-Adrenergic receptor blockade in chronic heart failure. Circulation 101: 558-569.

Cavusoglu E, Ruwende C, Chopra V, et al. 2006; Tissue inhibitor of metalloproteinase-1 (TIMP-1) is an independent predictor of allcause mortality, cardiac mortality, and myocardial infarction. Am Heart J 151: 1101.e1-8.

Cohn JN, Ferrari R, Sharpe N. 2000; Cardiac remodeling - concepts and clinical implications: a consensus paper from an international forum on cardiac remodeling. On behalf of an International Forum on Cardiac Remodeling. J Am Coll Cardiol 35: 569-582.

Deschamps AM, Spinale FG. 2006; Pathways of matrix metalloproteinase induction in heart failure: bioactive molecules and transcriptional regulation. Cardiovasc Res 69: 666-676.

Fedak PW, Verma S, Weisel RD, et al. 2005; Cardiac remodeling and failure: from molecules to man (Part I). Cardiovasc Pathol 14: $1-11$.

Fedak PW, Verma S, Weisel RD, et al. 2005; Cardiac remodeling and failure: From molecules to man (Part II). Cardiovasc Pathol 14: 49-60.

Fedak PW, Verma S, Weisel RD, et al. 2005; Cardiac remodeling and failure: from molecules to man (Part III). Cardiovasc Pathol 14: 109-119.

Fielitz J, Leuschner M, Zurbrugg HR, et al. 2004; Regulation of matrix metalloproteinases and their inhibitors in the left ventricular myocardium of patients with aortic stenosis. $J$ Mol Med 82: 809-820.

Li RK, Jia ZQ, Weisel RD, et al. 1996; Cardiomyocyte transplantation improves heart function. Ann Thorac Surg 62: 654-660.

Li RK, Jia ZQ, Weisel RD, et al. 1999; Smooth muscle cell transplantation into myocardial scar tissue improves heart function. J Mol Cell Cardiol 31: 513-522.

Li RK, Weisel RD, Mickle DA, et al. 2000; Autologous porcine heart cell transplantation improved heart function after a myocardial infarction. $J$ Thorac Cardiovasc Surg 119: 62-68.

Li H, Simon H, Bocan TM, et al. 2000; MMP/TIMP expression in spontaneously hypertensive heart failure rats: the effect of ACEand MMP-inhibition. Cardiovasc Res 46: 298-306.

Lukashev ME, Werb Z. 1998; ECM signalling: orchestrating cell behaviour and misbehaviour. Trends Cell Biol 8: 437-441.

Makino S, Fukuda K, Miyoshi S, et al. 1999; Cardiomyocytes can be generated from marrow stromal cells in vitro. J Clin Invest 103: 697-705.

Molina EJ, Palma J, Gupta D, et al. 2008; Improvement in hemodynamic performance, exercise capacity, inflammatory profile and left ventricular reverse remodeling after intra-coronary delivery of mesenchymal stem cells (MSC) in an experimental model of pressure overload hypertrophy. J Thorac Cardiovasc Surg 135: 292-299.

Nagaya N, Kangawa K, Itoh T, et al. 2005; Transplantation of mesenchymal stem cells improves cardiac function in a rat model of dilated cardiomyopathy. Circulation 112: 1128-1135.

Peterson JT, Li H, Dillon L, et al. 2000; Evolution of matrix metalloprotease and tissue inhibitor expression during heart failure progression in the infarcted rat. Cardiovasc Res 46: 307-315.

Polyakova V, Hein S, Kostin S, et al. 2004; Matrix metalloproteinases and their tissue inhibitors in pressure-overloaded human myocardium during heart failure progression. $J$ Am Coll Cardiol 44: 1609-1618.

Rajnoch C, Chachques JC, Berrebi A, et al. 2001; Cellular therapy reverses myocardial dysfunction. $J$ Thorac Cardiovasc Surg 121: 871-878.

Saito T, Kuang JQ, Lin CC, et al. 2003; Transcoronary implantation of bone marrow stromal cells ameliorates cardiac function after myocardial infarction. $J$ Thorac Cardiovasc Surg 126: 114-123.

Schwarz EJ, Reger RL, Alexander GM, et al. 2001; Rat marrow stromal cells rapidly transduced with a self-inactivating retrovirus synthesize L-DOPA in vitro. Gene Ther 16: 1214-1223.

Silva GV, Litovsky S, Assad JA, et al. 2005; Mesenchymal stem cells differentiate into an endothelial phenotype, enhance vascular density, and improve heart function in a canine chronic ischemia model. Circulation 111: 150-156.

Siwik DA, Chang DL, Colucci WS. 2000; Interleukin- $1 \beta$ and tumor necrosis factor- $\alpha$ decrease collagen synthesis and increase matrix metalloproteinase activity in cardiac fibroblasts in vitro. Circ Res 86: 1259-1265.

Spinale FG, Coker ML, Thomas CV, et al. 1998; Time-dependent changes in matrix metalloproteinase activity and expression during the progression of congestive heart failure: relation to ventricular and myocyte function. Circ Res 82: 482-495.

Suzuki K, Murtuza B, Suzuki N, et al. 2001; Intracoronary infusion of skeletal myoblasts improves cardiac function in doxorubicininduced heart failure. Circulation 104: I213-217.

Thomas CV, Coker ML, Zellner JL, et al. 1998; Increased matrix metalloproteinase activity and selective upregulation in LV myocardium from patients with end-stage dilated cardiomyopathy. Circulation 97: 1708-1715.

Tomita S, Mickle DA, Weisel RD, et al. 2002; Improved heart function with myogenesis and angiogenesis after autologous porcine bone marrow stromal cell transplantation. $J$ Thorac Cardiovasc Surg 123: 1132-1140.

Udelson JE, Konstam MA. 2002; Relation between left ventricular remodeling and clinical outcomes in heart failure patients with left ventricular systolic dysfunction. J Card Fail 8: S465-471.

Walther T, Schubert A, Falk V, et al. 2001; Regression of left ventricular hypertrophy after surgical therapy for aortic stenosis is associated with changes in extracellular matrix gene expression. Circulation 104: I54-58.

Wang X, Hu Q, Mansoor A, et al. 2006; Bioenergetic and functional consequences of stem cell-based VEGF delivery in pressureoverloaded swine hearts. Am J Physiol Heart Circ Physiol 290: H1393-1405.

Webb CS, Bonnema DD, Ahmed SH, et al. 2006; Specific temporal profile of matrix metalloproteinase release occurs in patients after myocardial infarction: relation to left ventricular remodeling. Circulation 114: 1020-1027.

Yan AT, Yan RT, Spinale FG, et al. 2006; Plasma matrix metalloproteinase-9 level is correlated with left ventricular volumes and ejection fraction in patients with heart failure. $J$ Card Fail 12: 514-519. 